\title{
Optical Energy Harvesting Materials
}

\author{
David L. Andrews \\ School of Chemical Sciences, University of East Anglia, \\ Norwich, NR4 7TJ, U.K.
}

\begin{abstract}
Optical energy harvesting signifies an integrated approach to the gathering of energy from light. The mechanisms which operate in the molecular apparatus of natural photosynthetic systems owe much of their efficiency to the highly localised pooling of energy - though that is not a feature that has been emulated in most artificial constructs. Recently, however, biomimetic energy pooling has become a theme of significant interest in the arena of photoactive materials, where applications are less restricted by a direct focus on the efficiency of solar energy harvesting. These materials present more immediate opportunities for implementation in devices for signal processing, optical computing and information technology (IT) applications. Exploiting advances in synthetic chemistry and laser photophysics, new energy pooling materials are designed to operate at much higher than ambient intensities of light, with operational characteristics geared to specific laser input wavelengths. In this chapter, it is shown how key principles learned from nature are being applied and extended in the design of optically nonlinear materials for energy pooling. Particular attention is focused on dendrimers, other multichromophore arrays, and rareearth doped materials.
\end{abstract}




\section{Introduction}

The modern technology of optical energy harvesting has a wide variety of operational principles, the main themes of which have evolved through pursuit of better control and economy in the global utilisation of solar energy. The concept of harvesting signifies an integrated approach to the gathering from natural or environmental resources, with centralised collection and subsequent distribution according to requirements. Over the years a plethora of schemes have been devised for the harvesting of energy, linking sustainability with economy and, increasingly, with green issues. Prominent amongst recent developments from traditional methods of environmental energy harvesting are as follows: (i) Devices for direct conversion of solar radiation to electric power - advanced antenna arrays; (ii) Energy production from the flow of moving water in streams, currents, or pipes - technology based on advanced piezoelectric materials; (iii) Micro-generators using the temperature differential between the ground and atmosphere - thermoelectric devices and materials; (iv) Micro-hydraulic transducers producing electrical energy from environmental mechanical oscillations - combining piezoelectric with micro-electromechanical systems (MEMS) technology. Many of the newer methods still under development signify relatively local, low-scale approaches to energy harvesting.

For the scale of the environmental resource it represents, and also the extent of its geographic availability, solar power easily outstrips its competition. Each year, in excess of $10^{24} \mathrm{~J}$ of solar energy impinges on the Earth. This exceeds by a factor of more than one hundred the next largest source of environmentally available power (wind energy, $10^{22} \mathrm{~J}$ ), while both are considerably in excess of a global demand that is not expected to reach $10^{21} \mathrm{~J}$ until the 2020's [1]. Natural photosynthesis represents a process whose emulation is an obvious target in energy harvesting. In fact most of the energy reaching the Earth's surface is infrared; $43 \%$ is visible and $4 \%$ ultraviolet. Infrared energy is only efficient at producing chemical change at very high intensities, well above those sustainably tolerable for biological systems. Visible light must be harvested for sustainable efficiency. However, simple consideration of the molecular energetics suggests that the mechanisms involved must be complex - and involve the collective action of two or more photons in some form of energy pooling. This is evident from the fact that, whereas photosynthesis generates oxygen through fission of water, the energy required to break any of their $\mathrm{O}-\mathrm{H}$ bonds $(4.8 \mathrm{eV}$, corresponding to an ultraviolet wavelength of $260 \mathrm{~nm}$ ) is beyond any one photon in the visible spectrum.

There are many well-established non-biological solutions to the energy problem, including photovoltaic action for the chemical storage of energy (e.g. photoelectrolysis) and photocatalytic use of metal-ligand complexes to lower the threshold energy for decomposition of water. Recently, for example, it has been shown that doping of indium tantalum oxide with nickel can reduce the band-gap to $2.3 \mathrm{eV}$, corresponding to a wavelength of $540 \mathrm{~nm}$ - though the mechanism is unclear and the quantum efficiency at $402 \mathrm{~nm}$ is only $0.66 \%$ [2]. Hybrid solar cells based on inorganic nano-rods interfacing semiconducting polymers appear to hold more promise, with prototype devices achieving efficiencies of $6.9 \%$ B]. However, as the detailed molecular structure and chromophore layout of the photosynthetic apparatus in a variety of living organisms have begun to emerge, new avenues of research are leading to significant advances in the modeling, synthesis and operation of distinctly 


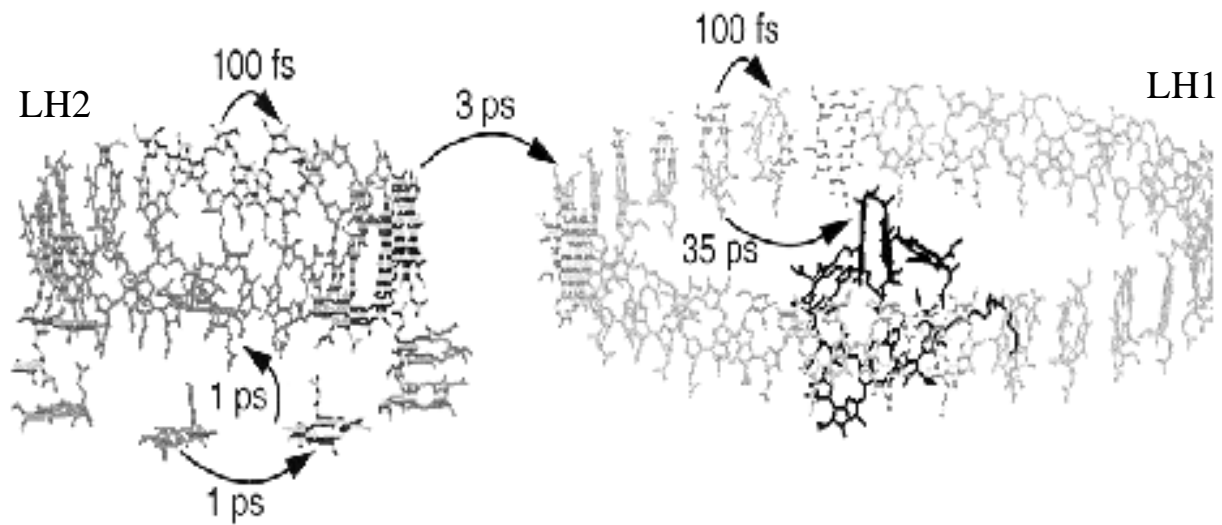

Figure 1: Key energy transfer processes in the light-harvesting complexes of purple photobacteria. Adapted from Ref. 5

biomimetic energy harvesting materials based on photon energy pooling. Whereas the purpose of man-made energy harvesting materials is not usually biosynthesis, both artificial and natural systems share a common need to capture and efficiently channel optical energy to a suitable site of repository, and crucial lessons can be learned from the structure and operation of photobiological systems [4].

\section{Precepts from photobiology}

In green plants, the photosynthesis of each elementary carbohydrate unit $\left(\mathrm{CH}_{2} \mathrm{O}\right)$ is achieved by two coupled redox reactions; one is the reduction of carbon dioxide, the other is the oxidation of water. It has long been known that each requires the energy of four photons for its completion. Natural photosystems generally comprise several non-covalently bound pigment-protein complexes. In each complex, the major protein component represents a superstructure within which the pigment molecules are held. In the visible region, the optical properties of such complexes are largely determined by the pigments, though the detailed spectral signatures of the latter are strongly influenced by their electronic surroundings.

Purple photosynthetic bacteria have light-harvesting systems whose structural and mechanistic properties [5-7] have been thoroughly characterized by means of ultrashort pulsed laser studies and X-ray protein crystallography. Here the photosynthetic unit comprises pigment-protein complexes in which the key light-harvesting pigment is bacteriochlorophyll. Two of the complexes, designated LH1 and LH2, form coplanar ring structures as schematically depicted in Fig. 1. In each circular aggregate, bacteriochlorophyll pigments take the form of dimers whose wavelength of maximum absorption is significantly modified by their protein environment. In common terminology, the bacteriochlorophyll dimer with an absorption maximum at $800 \mathrm{~nm}$ is referred to as B800. The complex LH2, on the left in Fig. 1, comprises both $\mathrm{B} 850$ (above) and $\mathrm{B} 800$ rings (below) whereas LH1, on the right, has a B880 ring. In fact many LH2 rings surround each LH1, as shown in Fig. 2. Together, these complexes deliver light energy to the reaction center ( $\mathrm{RC}$, shown at the center of LH1) where photosynthesis ensues. 


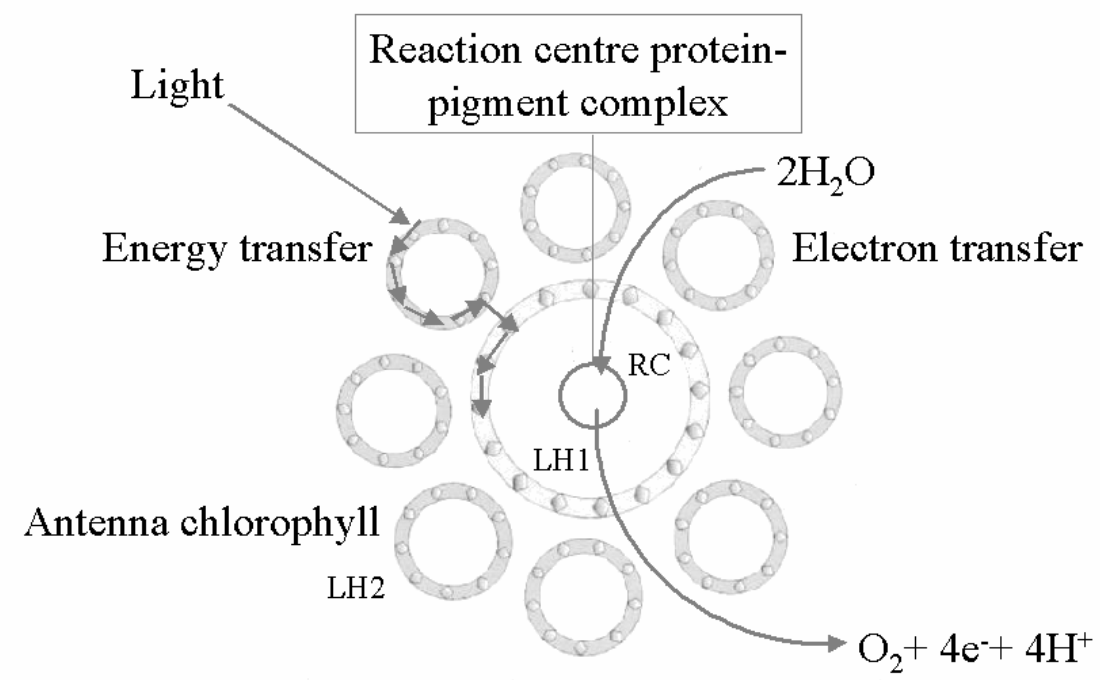

Figure 2: Energy flow in a bacterial photosystem for the oxidation of water. Adapted from Ref. 8.

A striking feature is the very rapid transfer of energy both within and between the light-harvesting arrays. In these multichromophore complexes, the operation of a spectroscopic gradient ensures that multistep energy migration is not simply a random walk; each transfer from one bacteriochlorophyll species to another absorbing at a longer wavelength signifies a small but significant loss of energy, ultimately manifest as heat. For any one transfer step, a key factor in determining its efficiency is the extent of overlap between the emission spectrum of the species from which energy departs (which in this connection assumes the role of donor - let us call it $A$ ) and the absorption spectrum of the species to which energy transfers (the acceptor, $B$ ). In any subsequent migration, where the erstwhile acceptor $B$ plays the role of donor, back-transfer to $A$ is largely inhibited by the much poorer overlap between the emission spectrum of $B$ and the absorption spectrum of $A$. For this reason the transfer of excitation energy towards the reaction center is favoured, a feature often referred to as channeling or funneling (the same principle also assists the oneway transfer of energy from carotenoids to chlorophyll species). Finally, at the reaction center, the energy of successively arriving photons is accumulated and serves to drive the redox half-reaction that initiates chemical photosynthesis.

Four key structural and mechanistic principles can be identified in the operation of such biological photosystems, and in generalised form many of the same principles inform the design of photoactive nanosystems and other light harvesting materials. These are as follows: (i) Efficient antennae are required; the chromophores responsible for photon absorption need broad and intense absorption bands (and also correspondingly strong emission bands, as will emerge below), i.e. strong oscillator strength; (ii) Excitation energy is conveyed through the system with high efficiency by a series of ultrafast steps. These usually involve the electromagnetic coupling mechanism known as resonance energy transfer, the 'resonance' signifying no energy losses during the actual transfer; (iii) Following each step in multistep energy migration, there may however be losses associated with intramolecular relaxation. Thus as the energy progresses through a series of different chromophores (or identical ones with increasing bathochromic shifts due to their electronic environment) a 
spectroscopic gradient serves to ensure directionality; (iv) The convergence of optically derived energy culminates in energy pooling, usually a process of upconversion (release of radiation with shorter wavelength) or physicochemical change - again, details are given below. To develop a more complete understanding of these factors we first turn to a more detailed appraisal of the photophysics involved in resonance energy transfer.

\section{Resonance energy transfer}

In considering the molecular mechanisms involved in each transfer step in a multi-step process, more than one kind of excitation transfer can be expected. The spectroscopic gradient principle alone strongly suggests the possibility of a difference in behavior when the donor and acceptor species are identical, as for example when we consider excitation transfer within one of the light-harvesting ring structures. Moreover, the possibility of close proximity between donor and (neighboring) acceptor within a ring structure clearly allows for much stronger coupling than could occur between well-separated chromophores. In the following, the main principle governing the transfer of excitation between wellseparated chromophores with different optical characteristics is first outlined, and the modified behavior observed in the identicalneighbor case is then identified.

The primary mechanism for energy transfer goes by a variety of names, each signifying a different aspect of the coupling: this incoherent, radiationless, Förster, or resonance energy transfer (RET) $[9,10]$ is also designated FRET in connection with the ensuing fluorescence [11]. It is a mechanism that mediates energy transfer in a wide variety of materials, including rare-earth doped crystals, Langmuir-Blodgett films and photoactive dendrimers, as well as multi chromophore arrays like the photosynthetic unit. The physical mechanism is dipole-dipole interaction between the excited donor and unexcited acceptor the two dipoles involved being transition dipoles for the decay of the donor and the excitation of the acceptor. The physical condition for this form of energy transfer is essentially weak coupling between the donor and acceptor species. In the modern theory based on quantum electrodynamics (QED), the coupling between the donor and acceptor transitions is calculated in terms of mediation by a short-lived, non-energy conserving, virtual photon - whose involvement can only be inferred; the energy transfer is radiationless for all practical purposes [12]. The QED theory in fact embraces both radiationless and longrange radiative energy transfer - the results are valid over the whole span of distances ranging from the nanoscale up to laboratory-scale distances and beyond [13-15].

Consider the pairwise transfer of excitation between two chromophore species $A$ and $B$. In this elementary process (which might be one RET component of a complex multichromophore mechanism) $A$ is designated the donor and $B$ the acceptor - though each may adopt another role subsequent transfer events. Specifically, let it be assumed that prior excitation of the donor generates $A^{*}$, in which it occupies an excited state $S_{n}$. Onward progress of the energy is then accompanied by donor decay to a manifold of states associated with the ground electronic state $S_{0}$. Acquiring the energy, the acceptor $B$ undergoes transition from its ground state $S_{0}$ to its excited manifold $S_{n}$, as illustrated in Fig. 3. (The excited acceptor, $B^{*}$, subsequently decays either in a further transfer event or by another means such as fluorescence.) 


$$
A^{*}+B \stackrel{\text { RET }}{\rightarrow} A+B^{*}
$$

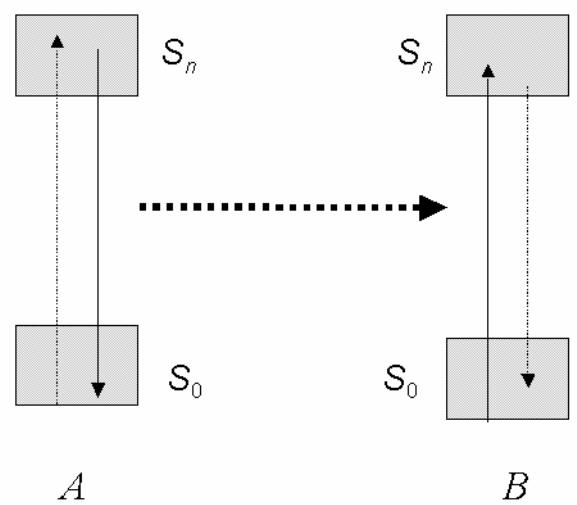

Figure 3: Energetics in one stage of resonance energy transfer. In the QED theory based on virtual photon coupling, donor decay may precede or follow (with ultrafast rapidity) the acceptor excitation.

Restricting consideration to donor-acceptor separations $R$ substantially smaller than the wavelengths of visible radiation, the theory gives the following expression for the rate of pairwise energy transfer $w$, applicable for systems where the host for the donor and acceptor has refractive index $n$ :

$$
w=\frac{9 \kappa^{2} c^{4}}{8 \pi \tau_{A} n^{4} R^{6}} \int F_{A}(\omega) \sigma_{B}(\omega) \frac{d \omega}{\omega^{4}} .
$$

In this expression, $\kappa$ is an orientation factor given later in $(3) ; F_{A}(\omega)$ is the normalized spectrum of donor fluorescence; $\sigma_{B}(\omega)$ is the linear absorption cross-section of the acceptor and $\omega$ the optical frequency in radians per second $(=2 \pi v$, with in $\mathrm{Hz}) ; c$ is the speed of light. An immediately evident implication of the result is that efficient energy transfer demands significant overlap of the emission spectrum for $A$ with the absorption spectrum of $B$. (A similar principle operates in microwave energy transfer, whose efficiency depends on the corresponding overlap of Fourier mode components). Also featuring in the above result is $\tau_{A}$, the radiative decay lifetime of the donor, related to the measured fluorescence lifetime $\tau_{\text {fluor }}$ through $\tau_{\text {fluor }}=\eta \tau_{A}$, where $\eta$ is the fluorescence quantum yield. An alternative, exactly equivalent expression for the rate of resonance energy transfer is as follows:

$$
w=\frac{2 \kappa^{2}}{3} \frac{1}{\tau_{A}}\left(\frac{R_{0}}{R}\right)^{6}
$$

Here, $R_{0}$ is the critical or Förster radius, calculable from the overlap integral of the donor fluorescence and acceptor absorption spectra; typical values of the Förster radius range over a few nanometers.

Apparent in both (1) and (2), the inverse sixth power dependence on donor-acceptor separation $R$ is a well-known characteristic of dipole-dipole energy transfer. It determines the fact that, with intermediary donor/acceptor species suitably positioned and electronically distinct, any process resulting in 
the overall migration of excitation over distances beyond the Förster radius will mostly operate through a series of short hops rather than one long hop (see below regarding exciton propagation). The $\kappa$ factor in the energy transfer rate equation (which is, in fact, identical in form to the orientational dependence in static dipole coupling) depends on the orientations of the donor and acceptor, both with respect to each other and with respect to their mutual displacement unit vector $\widehat{\mathbf{R}}$, as follows:

$$
\kappa=\left(\hat{\mu}_{A} \cdot \hat{\mu}_{B}\right)-3\left(\hat{R} \cdot \hat{\mu}_{A}\right)\left(\hat{R} \cdot \hat{\mu}_{B}\right)
$$

Here, for each molecule, $\hat{\mu}$ designates a unit vector in the direction of the appropriate transition dipole moment. Unfavourable orientations can reduce the rate of energy transfer to zero; others, including many of those found in nature, will optimise the transfer rate. The angular disposition of chromophores is therefore a very important facet of energy transfer, and one that invites careful consideration in the design of energy harvesting materials. In correcting a common misconception, note that transfer is not necessarily precluded when the transition moments lie in perpendicular directions - provided that neither is orthogonal to $\mathbf{R}(=R \widehat{\mathbf{R}})$.

The dipole-dipole coupling which leads to the foregoing results is in certain cases not applicable, as for example if either the donor or acceptor transition is electric dipole (E1)-forbidden or exceptionally weak. Then, the mechanism for energy transfer can involve the electric quadrupole moment (E2), higher electric multipoles (En) or even magnetic multipoles ( $\mathrm{M} n)$, in each case leading to an orientation and distance dependence of a different form. In the most common case of predominantly electric coupling, if (En) and (Em) are the leading non-zero moments of the donor and acceptor, the distance dependence takes the form $R^{-2(\mathrm{n}+\mathrm{m}+1)}[16,17]$. Observations of energy transfer associated with dipole-forbidden interactions often reveal a degree of efficiency that precludes explanation simply on the basis of higher-order multipole coupling, however. The Dexter exchange mechanism [18] is based on the premise that when the electron distributions of the donor and acceptor are close enough to overlap strongly, the energy of electronic excitation can pass directly to the acceptor, essentially channeled by the overlapping electron clouds. As such it is a mechanism that comes into play only at very short distances where it exhibits an exponential dependence on $R$, reflecting a typical asymptote for the wavefunctions of the molecular orbitals. It is not always appreciated that the Dexter mechanism can operate over such short distances both for dipoleforbidden and also dipole-allowed transitions; for an account of how the Dexter and Förster mechanisms seamlessly merge for the dipole-allowed case, see Scholes and Ghiggino [19]. When donor and acceptor chromophores are electronically distinct, however, one should account for dipole-forbidden transitions in terms of higher multipoles.

It is known that the dielectric properties of the medium within which energy transfer takes place (the protein matrix in photosynthetic systems, for example) exercises a considerable influence on the transfer efficiency, beyond the refractive dependence exhibited in the Förster formula (1) [20]. Where electronically prominent chromophore species intervene between donor and acceptor groups, the detailed understanding of such an influence has to be sought at the molecular level. For example, one obvious factor in determining the efficiency of excitation transfer is possible mediation of the transfer through 
the electronic involvement of a bridge or other suitable placed species. Work on rigidly linked bichromophore and multichromophore molecules has given conclusive evidence that, under certain conditions, a through-bond superexchange interaction can dominate intramolecular energy transport [21-23]. A recent analysis based on molecular QED has examined a number of the broader issues associated with the electronic influence of a neighbouring chromophore on resonance energy transfer, showing how it can be tailored to effectively 'switch on' the energy transfer process under circumstances where energy transfer is normally forbidden by orientationaleffects or symmetry grounds [24].

In the case of identical chromophores, closely neighbouring but electronically distinct, differences arise through the possible formation of excitons. It is as well to make clear the meaning of the term exciton in the present context since elsewhere it is employed with a wide variety of connotations - even including the single donor- or acceptor-localised excitations discussed above. In common with much of the recent literature on energy harvesting, however, exciton here denotes a non-propagating, delocalised electronic state associated with the quantum interference of separate chromophore states. At simplest, the excited states of any isolated pair (comprising a strongly coupled donor and acceptor) can mix and form a delocalised excited state split by twice the coupling energy. With such excitonic states one can no longer specify the molecular location of the electronic excitation; by extension, in light-harvesting complexes comprising large numbers of equivalent chromophores, excitons can spread over several equivalent donor/acceptor species and be associated with numerous, closely separated energy levels. Indeed, the separation between the lowest levels affords a useful means of gauging the extent of exciton delocalisation 25]. Although any local disorder can substantially compromise the extent of excitonic delocalisation and also any superexchange coupling, a surprising finding in connection with B800-B850 coupling was that site disorder can be responsible for a more rapid transfer of excitation than would occur in its absence 26]. The multiplicity of the associated exciton splitting enhances spectral overlap in multichromophore arrays and thereby accelerates energy transfer. Thus, in natural light-harvesting complexes the ring structures, which provide symmetry in the pigment arrays, enhance absorption and lead to those arrays as a whole acting as nanoscale energy traps. The mechanistic interpretations of many bacterial and other photosynthetic systems are based on this premise - see Refs [27-29].

\section{Dendrimers}

Following several decades in which the science of energy-harvesting materials was largely pursued through the development and optimisation of photovoltaic cells, the arrival of dendrimeric macromolecules has heralded a new opportunity for achieving more closely biomimetic methods for light harvesting [8]. Dendrimers are multi-branched nanoscale structures, with essentially fractal geometry and a large number of chemically similar chromophores amongst which energy can transfer; as such they are also known as nanostars or functional cascade molecules [30,31]. Much current work is directed towards the use of photoactive dendrimers - not only as model systems for photosynthetic light harvesting, but also as light-emitting diodes, fluorescent sensors, frequency conversion materials and other photonic devices. Future advances may lead towards more directly biomimetic energy harvesting systems. 


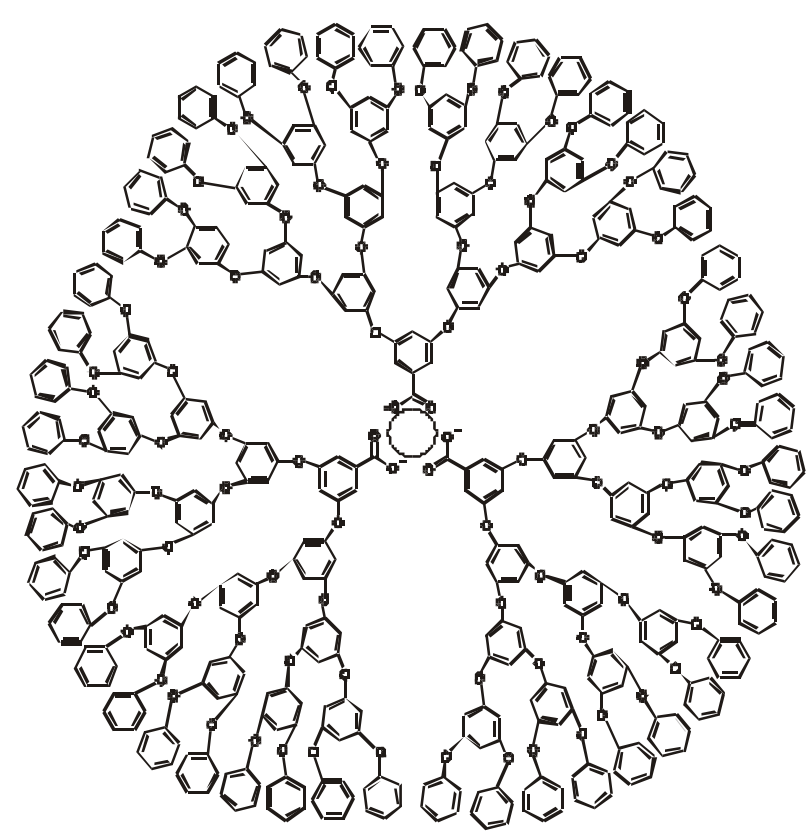

Figure 4: Polyphenylether dendrimer with a photoactive trap.

One of the objectives is to achieve, through funneling and trapping, the kind of energy pooling that characterizes biological systems, where the energy of more than one photon is accumulated in a trap - a topic that is to be revisited later in this chapter.

In the typical example shown in Fig. 4, some of the features also common to natural photosynthetic systems are immediately apparent: principally, the circularly symmetric ring-like array of chromophores about a central excitation trap. However, not only these structural motifs are important; it is also highly significant that the chromophore units are electronically distinct, here a result of the alternate 1,3,5-substituent linkage pattern on each benzene ring. Again, the ether linkage has the effect of electronically isolating the phenyl chromophores; ethynyl linkages are also commonly employed in such dendrimers to similar effect, as detailed theory [30,32] and spectroscopic evidence [33] has shown. As a result there is negligible through-bond coupling between the chromophore units, and transfer of excitation between them essentially takes the form of dipole coupling.

In any dendrimer the synthetic route gives successive generations, each expanded by the peripheral addition of further functional groups. The addition of further chromophores adds to the photon capture efficiency of the dendrimer, though the associated generation-dependence in the distribution of interchromophore distances can result in a reduction in efficiency of energy transfer [34]. Clearly the central unit, even when of chemically identical form to the dendrite arms, differs in its electronic environment from those other chromophores - equally, amongst those, the peripheral chromophores are in a still different environment. This can be played to advantage; in ideal cases the associated shifts in the corresponding absorption and fluorescence spectra can foster exactly the kind of spectroscopic or energy gradient observed in natural systems. This principle has been neatly illustrated in work on perylenefunctionalised phenylacetylene dendrimers, where the directionality of energy 
flow from antenna chromophores to the core is a hundred times greater than in the analogous structure with dendrons all of equal length [35,36]. As observed in section 2 such an energy funnel represents a combination of structural and spectroscopic elements together directing energy towards the core, the latter acting as the trap [37,38]. The efficient unidirectional transfer of energy within a dendrimeric frame towards a core chromophore was in fact first reported only as recently as 1994 [39]. A new development in this area has been the design of dendrimers whose internal cavities host smaller dye molecules. Exploiting not only intramolecular but also intermolecular (dendrimer - dye) energy transfer, extremely harvesting efficiencies (estimated at around 80\%) have recently been reported for eosin embedded in a dendrimer with 65 chromophore groups of four different types [40].

Although not strictly dendrimeric, other light-harvesting materials with analogous structural motifs have also been investigated. A molecular square, twenty-chromophore unit has recently been constructed by use of metal ion directed self-assembly methods, and shown to exhibit biomimetic energy transfer from outer to inner (pyrene to perylene) chromophores [41]. Closer to nature, multi-porphyrin systems in particular appear to offer considerable promise [42]. Here, for example, it has been shown that excitation transfer, between a zinc porphyrin acting as donor and its free base counterpart as acceptor, can be significantly enhanced by exploiting suitable bridging chromophores [43]. As with the dendrimers, these structures are designed to obviate transfer be tween donor and acceptor species through charge conjugation or wavefunction overlap. Other recent work has forged a synthetic and conceptual link in this area, through the synthesis and characterisation of multi porphyrin arrays based on a phenylethynyl proto-dendrimeric framework [44]. A striking success is an artificial photosynthetic antenna-reaction center complex comprising four zinc tetraarylporphyrins covalently linked, through phenylethynyl dendrites, to a free base porphyrin-fullerene acting as a 'reaction center'. Following photoexcitation of the peripheral zinc porphyrin (antenna), energy migrates to the central zinc porphyrin (donor) from which it transfers to free base porphyrin (acceptor), initiating electron transfer to the fullerene (reaction centre). In this system, the charge-separated excited state is generated with an impressively high quantum yield of 0.90 , based on the light absorbed by the zinc porphyrin antenna $[45,46]$.

\section{$5 \quad$ Rare-earth materials for energy pooling}

It may at first sight be surprising to discover several of the mechanistic principles discussed in the foregoing dendrimeric materials operating in the field of solid-state crystal optics (indeed, recognition of this has led to some highly innovative work on advanced energy pooling nanomaterials, to be discussed later in this chapter). Conventional energy transfer does to some extent modify the operating characteristics of laser crystals; in essence, excitation hopping competes with radiative stimulated emission as a channel of decay for electronically excited ions. However in laser systems other novel forms of energy transfer can occur - in particular, processes of concerted energy pooling become possible.

The pooling that features in photobiological systems is associated with the eventual collection of energy acquired from photons which have been absorbed at various earlier times; moreover, various chromophore species experience electronic excitation and subsequent decay in the process of energy 
migration to the reaction center. However, the much higher levels of photon flux in laser systems makes it possible to pool the energy of photons which arrive together (within an ultrashort timescale). Consequently, the time-energy uncertainty principle allows intermediaries in the process of energy transport to undergo transitions to extremely short-lived, non-energy conserving virtual states. In this respect, the process has a similar basis to two-photon absorption, and indeed most other forms of optical nonlinearity. Experimentally, such energy pooling becomes manifest in certain observations of blue-shifted fluorescence; notably, it has been shown that rare-earth (lanthanide) doped crystals display the effect at high levels of optical excitation. In general, this fluorescence owes its origin to energy transfer mechanisms involving three chromophore/fluorophore sites, with two acting as donors and one as acceptor [47-49]. Exploiting the effect, it is possible to tailor such materials specifically for stepwise or sequential laser frequency upconversion.

The reason for rare-earth ions to be so favoured in this connection is that they display strong $f-f$ (core $f$-electron) transitions associated with a redistribution of charge sufficiently close to the nucleus that the ionic environment exercises relatively little influence. As a consequence, their absorption and fluorescence spectra contain highly discrete, characteristic and well-resolved lines, with many falling in wavelength regions eminently suitable for laser excitation [50-52]. It should be emphasised that the materials under discussion here operate quite differently from conventional nonlinear optical crystals (where off-resonance input photons directly engage in pairwise or higher order coupling, not involving any optical excitation of the material). By contrast, energy pooling processes are non-parametric and generally involve dissipative losses; indeed, associated phonon generation can expedite transfer by modifying the selection rules. The features such materials have in common with those discussed previously are largely those bulleted at the end of Section 3 , here to be understood in an atomic context. Specifically, there must be high efficiency in photoabsorption, energy transfer and energy pooling; only the spectroscopic gradient is less relevant - as fewer transfer steps are involved.

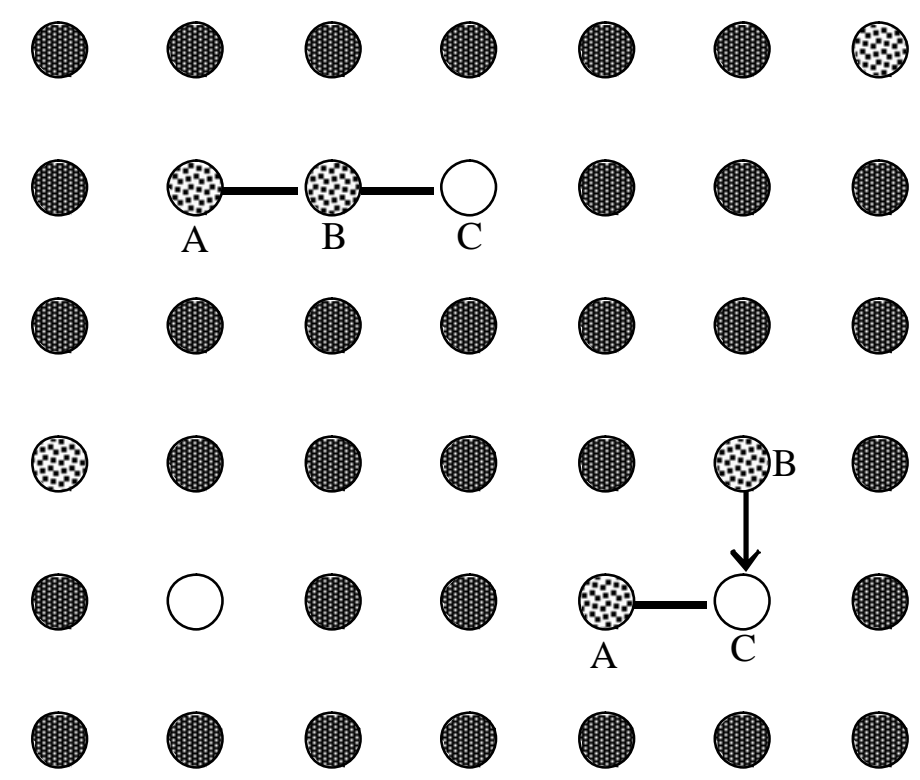

Figure 5: Accretive (upper left) and cooperative (lower right) energy pooling. Dotted circles - excited donors: open circles - acceptors: dark circles - ground state species. 


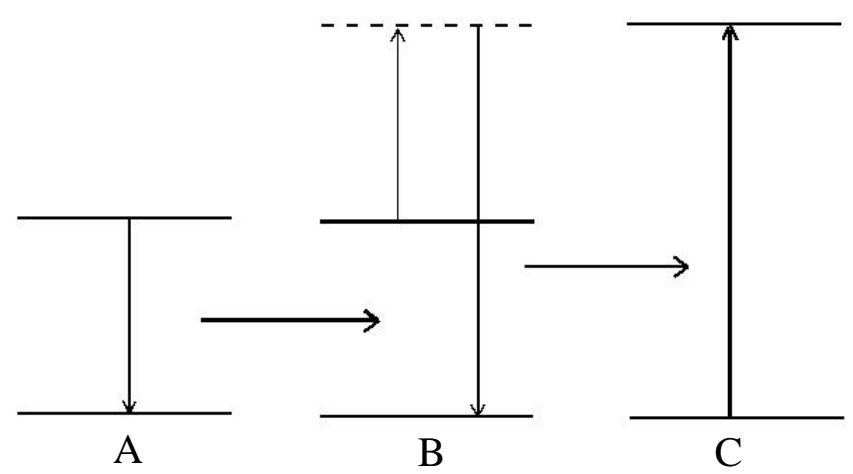

Figure 6: Energetics of excitation pooling: accretive mechanism. Horizontal lines denote electronic levels; the dotted line a virtual intermediate state. Each vertical arrow

designates an electronic transition associated with single-photon selection rules.

In detail, the mechanisms which operate in rare earth energy pooling materials entail resonance energy transfer from two initially excited ionic fluorophore donors $A$ and $B$ (which may, but need not, be chemically identical), to other ion acceptors $C$. Two fundamentally different mechanisms can mediate the effect. In one mechanism, illustrated top left in Fig. 5, energy transfer involves routing from $A$ to $C$ via $B$, at which intermediate point the additional excitation energy of $B$ is acquired. The sum energy thereby delivered to $C$ accomplishes the excitation of $C$, whose ensuing fluorescent decay produces the observed, up-converted radiation. The pooling of energy at $C$, which should not be thought of as a two-step process since energy need not be conserved before its completion, has been designated an accretive mechanism with due regard to the nature of the intermediary role of $B$ [53]. The overall process is still regarded as proceeding in stepwise fashion because the donor excited sates have a finite lifetime before energy transfer, as does the acceptor excited state prior to its fluorescent decay. A second mechanism, by means of which energy is deposited at $C$ directly from the two donors, is termed cooperative [54]; it is depicted in the lower right of Fig. 5. The general characteristics of both mechanisms have been determined through a detailed QED appraisal [55].

As illustrated in Fig. 6, accretive pooling requires the donor decay to satisfy both single- and two-photon selection rules. In the cooperative mechanism, Fig. 7, single-photon transitions characterise each donor decay and

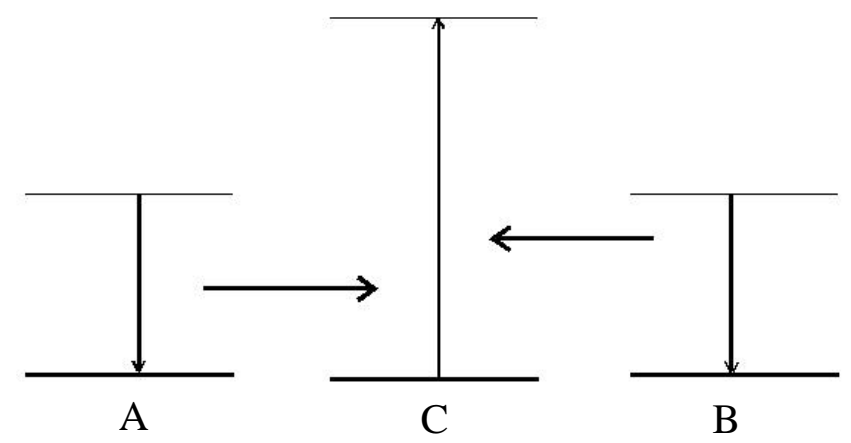

Figure 7: Energetics of excitation pooling: cooperative mechanism. Horizontal lines denote electronic levels; the dotted line a virtual intermediate state. Each vertical arrow designates an electronic transition associated with single-photon selection rules. 
excitation of the acceptor follows two-photon selection rules. To observe either form of such energy pooling, in a system accommodating donor and acceptor species appropriate to the laser input, it is necessary to furnish the necessary levels of donor excitation. The specific requirement here is for pairs of donors, $A$ and $B$, to be excited through conventional single-photon absorption - so that during a certain period, they are simultaneously in excited states. In the simplest practical case, the donor species are identical and both become excited by the same input radiation. It has long ago been established that two-site single-photon absorption is not intrinsically less probable than the more familiar single-site process of two-photon absorption [56] - in general, pulsed laser irradiation can readily afford the means of establishing the necessary level of donor excitation for both cooperative and accretive energy pooling. In the optically primed system (i.e. following pump excitation) the relative number densities of excited donors and acceptors is therefore a significant determinant of mechanism, coupled with the different optical selection rules that operate for each mechanism.

In the most general case, $A, B$ and $C$ may be three chemically different species (or the transitions involved in them may differ even when there is atomic equivalence). Then, in determining the efficiency of energy pooling in a doped crystal it is necessary to consider all permissible ion positions on the lattice, and also the number of ions $A, B$, and $C$, residing in their appropriate initial electronic states, per unit cell. If these are written as $C_{X}$ for species $X$, then the appropriate concentrations, in a cubic crystal with cell length $a$, are expressible as $c_{X}=a^{-3} C_{X}$. Where the optical selection rules permit only one mechanism to operate the rate of energy pooling, for each triad combination of $A, B$, and $C$ ions, depends on the inverse sixth powers of two inter-ionic distances. These are independent of each other - though calculations must discount any triad where two or three ions share identical lattice sites. Allowing for all these restrictions, and assuming random positioning of the salient ions, the lattice sums that feature in the ensemble rates result in the following energy harvesting efficiency factors [55]:

$$
\begin{aligned}
& \sum_{\text {lattice }} R_{A B}^{-6} R_{B C}^{-6} \rightarrow \eta C_{\mathrm{A}} C_{\mathrm{B}}^{2} C_{\mathrm{C}} a^{-12}=\eta c_{\mathrm{A}} c_{\mathrm{B}}^{2} c_{\mathrm{C}} \quad \text { (accretive) }, \\
& \sum_{\text {lattice }} R_{A C}^{-6} R_{B C}^{-6} \rightarrow \eta C_{\mathrm{A}} C_{\mathrm{B}} C_{\mathrm{C}}^{2} a^{-12}=\eta c_{\mathrm{A}} c_{\mathrm{B}} c_{\mathrm{C}}^{2} \quad \text { (cooperative) } .
\end{aligned}
$$

Here $\eta$ is a structure-dependent numerical factor, here taking the cubic lattice value, $\eta=64.39$. The lattice weightings thus signify concentration factors for each of the participating ions in the necessary initial states. Where selection rules permit both accretive and cooperative pooling to occur (which is possible when inversion symmetry is absent), quantum interference terms have also to be accommodated in equations for the rate of energy harvesting. For ions randomly disposed on a cubic lattice, rate contributions from such interferences are just as important as the 'pure' accretive or 'pure' cooperative kind. In connection with conventional (single-donor) energy transfer, calculations based on this method have been applied extensively to crystal systems [57,58], with some work also accounting for higher multipole couplings and the exchange interaction $[59,60]$. The corresponding extensions to multiphoton energy pooling remain the subject of active research. 
Examples of twin-donor energy pooling in up-conversion are rife, and not necessarily limited to systems containing more than one species of rareearth ion. The occurrence of such a process was, for example, first identified in systems involving a concerted action amongst three electronically excited $\operatorname{Pr}^{3+}$ ions, in which two ions decay and the third is further excited through the acquisition of their combined energy [61,62]. It has been shown that an isoionic process also accounts for green and red emissions from $\mathrm{Er}^{3+}$ in fluoritetype crystals [63].

\section{Energy pooling in multichromophore arrays}

Much recent work on energy pooling has been directed towards nanoscale molecular emulation of the up-conversion principles that operate in rare earth materials. In particular, dendrimeric and other still more closely biomimetic light-harvesting arrays offer great scope for technological applications. In this connection, theoretical work is progressing hand in hand with current synthetic efforts, each informing the other; in both areas the difficulties are considerable, yet good progress is being made. Part of the challenge lies in dealing with the complexities associated with energy transfer in systems with intricate molecular architecture [64]. For natural systems, revelation of the light-harvesting structures has enabled theory to home in on the interplay of the relevant electronic properties, a carte blanche where artificial systems are concerned.

One of the first examples of energy pooling was the observation of two-photon excitation energy transfer in a trichromophoric system, as illustrated in Fig. 8 [65]. Here, antenna species were excited with an optical frequency off-resonant with respect to direct one- or two-photon excitation of the central stilbene, which nonetheless exhibited energy uptake through $E / Z$-isomerisation. There is also some evidence to suggest the operation of such mechanisms in photoresponsive dendrimers, particularly those based on an azobenzene core with a pair of benzyl aryl ether dendrites. Here the energy required to effect the observed cis-trans isomerization of the core appears to be only consistent with the pooling of excitation from two of the chromophores [66,67]. Meanwhile, other dendrimers, based on (diphenylamino)stilbene, have been shown to exhibit exceptionally high rates of two-photon absorption [68].

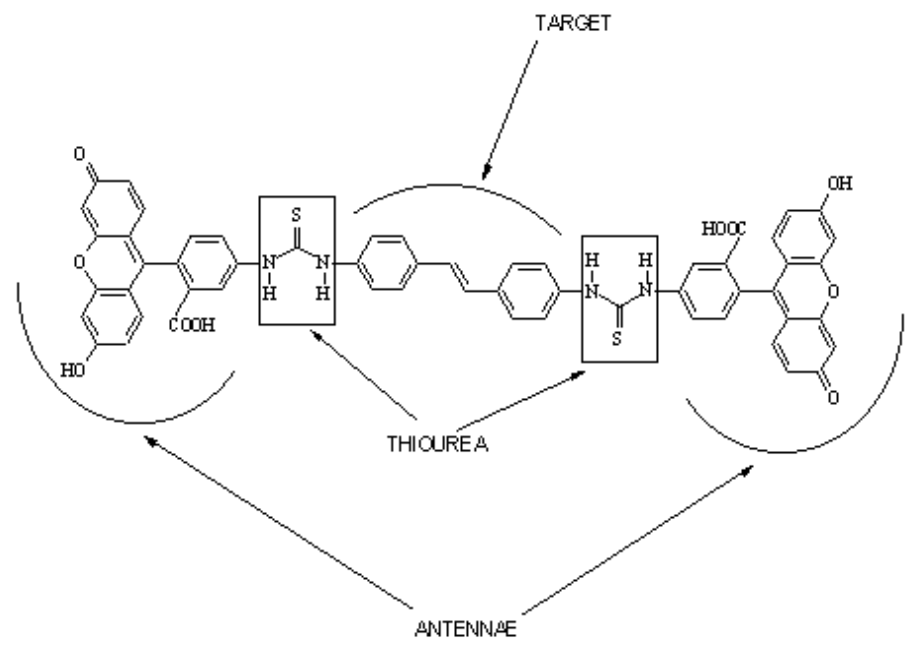

Figure 8: Twin-donor cooperative energy transfer. 


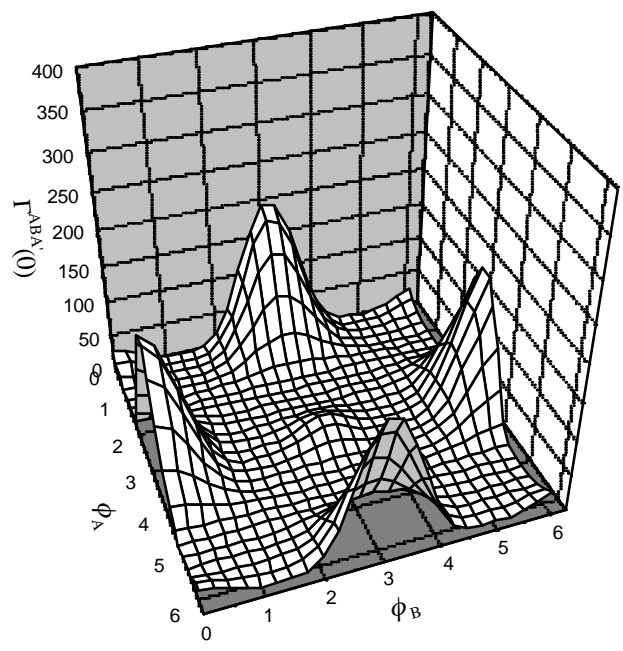

Figure 9: 3D plot showing variation of the rate of trichromophore energy pooling with angles $\phi_{\mathrm{A}}$ and $\phi_{\mathrm{B}}$, in a molecular architecture favouring the cooperative mechanism.

For multichromophore arrays, the complexity of energy pooling calculations increases considerably, especially in connection with the selection rules and orientational factors [69]; indeed, for their full analysis, multidimensional optimization routines are required. By way of illustration, Fig. 9 shows a three-dimensional plot of the variation of efficiency for cooperative transfer of energy in a twin-donor system, as a function of the angles made by the transition dipole moments of the two donors, $\phi_{A}$, and that of the acceptor, $\phi_{B}$, relative to the system axis. Here it is assumed that the acceptor is situated directly in-between the two donors, as in the system shown in Fig. 8. However, for simplicity, it is assumed that all three transition moments are coplanar and that both donor moments are collinear [70]. Even such results apply just for one of the two contributing mechanisms; the accretive mechanism requires a similar appraisal.

Recent theory has highlighted the need to simplify conceptualisation of multichromophore energy pooling, expedited through the application of a new interaction-pair terminology [71]. This formalism can obviate conceptual and semantic problems in the correct description of multichromophore (or in general, multi-center) interactions. In connection with triple-donor pooling, a schematic depiction as in Fig. 10 accommodates the overall light-harvesting operation of a model dendrimer. It establishes the fact that four mechanisms,
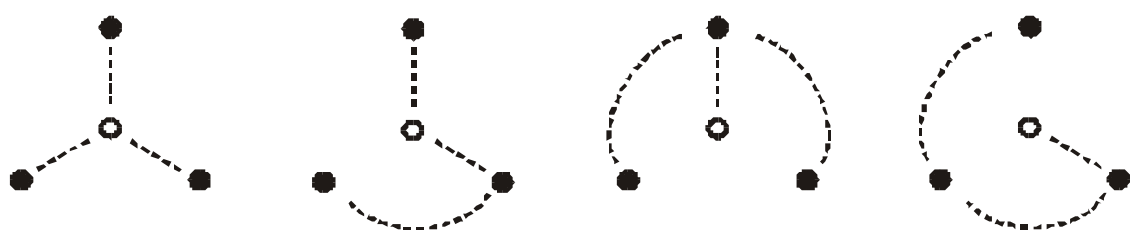

Figure 10: Depiction of energy transfer routes for a triply-excited donor array. 
with varying degrees of accretive and cooperative character, may operate unless one or more is precluded by the selection rules or by the geometry [72]. However, such illustrations fail to reveal a significant feature that arises where two or more mechanisms do come into play. It needs to be emphasised that the rate of energy harvesting generally has not only contributions associated with each route individually, but also others resulting from their quantum interference, which may signify either an enhancement or a reduction in the overall energy harvesting efficiency. Only detailed QED calculations reveal the extent to which the different quantum channels are interlaced. Moreover, for a multichromophore structure, the calculation of interference terms has to accommodate all possible chromophores as intermediaries. Another quantum feature concerns the time-ordering of the elementary interactions. Fig. 10 designedly omits any arrowheads suggesting directions or orderings for the virtual photon couplings from which each mechanistic pathway is constructed, because all temporally distinct orderings must be accommodated in the theory. A simple consequence of the time-energy uncertainty principle, proper accommodation of this feature is necessary in order to correctly derive the form of the electronic tensors for the chromophores involved. Through detailed calculation it emerges that these tensors are closely linked to those which characterize multiphoton absorption and scattering.

\section{The future of energy pooling}

Optical energy harvesting is an area of science characterised by an almost unparalleled degree of interdisciplinarity. Inspired by discoveries in photobiology, operating on principles from materials scie nce, spectroscopy and both quantum and nonlinear optics, this rapidly developing subject enjoys the unique facility to accommodate their insights, and to exploit their design strategies for burgeoning technological applications. In this review, attention has focused particularly on the principles and mechanisms of biological photosynthesis, photoactive dendrimers and other multichromophore arrays, and also rare-earth materials. While much research effort is directed towards devising more and more closely biomimetic analogues of natural photosynthetic light-harvesting arrays, other important developments are taking place in the pursuit of materials to exploit optical nonlinearity for energy pooling under conditions of intense optical irradiation.

Much of the recent work on theory has aimed to establish the underlying principles and links between the modes of operation of such energy harvesting systems. Key factors here include the chromophore layout and geometry, the electronic structure and optical selection rules. The fundamental pair-coupling nature of energy pooling becomes manifest in the detailed form of the material parameters, and especially in the interconnection between the diverse quantum channels that can mediate multiphoton excitation trapping [73]. Current work in this area is aiming to fully elicit the significance and role of electronically delocalised excitons in multichromophore arrays, with particular regard to exclusively exciton-mediated channels for energy transfer $[74,75]$. A complete assessment of the interplay between all of these factors will prove crucial for the future design and optimisation of new photoactive materials. 


\section{Acknowledgements}

I am greatly indebted to Werner Weiglhofer, whose kind invitation to write this chapter was characteristically followed up with many helpful and insightful comments. His wisdom will be greatly missed. Thanks are due to Akhlesh Lakhtakia for much other valuable feedback - and also to Robert Jenkins, at the University of East Anglia (UEA), who in addition produced several of the diagrams. Research in the QED group at UEA is funded by the Engineering and Physical Sciences Research Council.

\section{References}

[1] S.F. Baldwin, Renewable energy: Progress and prospects, Phys Today 55 (4) (2002), 62-67.

[2] Z. Zou, J. Ye, K. Sayama and H. Arakawa, Direct splitting of water under visible light with an oxide semiconductor photocatalyst, Nature $\mathbf{4 1 4}$ (2001), 625-627.

[3] W.U. Huynh, J.J. Dittmer and A.P. Alivisatos, Hybrid nanorod-polymer solar cells, Science 295 (2002), 2425-2427.

[4] P. Ball, Natural strategies for the molecular engineer, Nanotechnology 13 (2002), R15-R28.

[5] R. van Grondelle and O.J.G. Somsen, Excitation energy transfer in photosynthesis, Resonance energy transfer (D. L. Andrews and A. A. Demidov, eds), Wiley, New York, NY, USA, 1999.

[6] X. Hu and K. Schulten, How Nature harvests sunlight, Phys Today 50 (8) (1997), 28-34.

[7] K. Schulten, From simplicity to complexity and back: Function, architecture, and mechanism of light harvesting systems in photosynthetic bacteria, Simplicity and complexity in proteins and nucleic acids $(\mathrm{H}$. Frauenfelder, J. Deisenhofer and P. G. Wolynes, eds) Dahlem University Press, Berlin, Germany, 1999.

[8] A. Adronov and J. M. J. Fréchet, Light-harvesting dendrimers, Chem Commun (2000), 1701-1710.

[9] V. M. Agranovich and M. D. Galanin, Electronic excitation energy transfer in condensed matter, Elsevier/North-Holland, Amsterdam, The Netherlands, 1982.

[10] B. W. van der Meer, J. Coker III, and S.-Y. S. Chen, Resonance energy transfer: theory and data, VCH, New York, NY, USA, 1982.

[11] P. R. Selvin, The renaissance of fluorescence resonance energy transfer, Nature Struct Biol 7 (2000), 730-734. 
[12] D. L. Andrews, A unified theory of radiative and radiationless molecular energy transfer, Chem Phys 135 (1989), 195-201.

[13] D. L. Andrews and G. Juzeliunas, Intermolecular energy transfer: retardation effects, J Chem Phys 96 (1992), 6606-6612.

[14] D. L. Andrews and P. Allcock, Bimolecular photophysics, Chem Soc Rev 24 (1995), 259-265.

[15] G. Juzeliunas and D. L. Andrews, Quantum electrodynamics of resonance energy transfer, Adv Chem Phys 112 (2000), 357-410.

[16] G. D. Scholes, A. H. A. Clayton and K. P. Ghiggino, On the rate of radiationless intermolecular energy-transfer, J Chem Phys 97 (1992), 74057413.

[17] G. D. Scholes and D. L. Andrews, Damping and higher multipole effects in the quantum electrodynamical model for electronic energy transfer in the condensed phase, J Chem Phys 107 (1997), 5374-5384.

[18] D. L. Dexter, A theory of sensitized luminescence in solids, J Chem Phys 21 (1953), 836-850.

[19] G. D. Scholes and K. P. Ghiggino, Electronic interactions and interchromophore excitation transfer, J Phys Chem 98 (1994), 4580-4590.

[20] C.-P. Hsu, G. R. Fleming, M. Head-Gordon and T. Head-Gordon, Excitation energy transfer in condensed media, J Chem Phys 114 (2001), 3065-3072.

[21] K. P. Ghiggino, E. K. L. Yeow, D. J. Haines, G. D. Scholes and T. A. Smith, Mechanisms of excitation energy transport in macromolecules, $J$ Photochem Photobiol A: Chemistry 102 (1996), 81-86.

[22] E. K. L. Yeow, D. J. Haines, K. P. Ghiggino and M. N. Padden-Row, Electronic energy transfer in multichromophoric arrays. A sequential and superexchange dynamics study, J Phys Chem A 103 (1999), 6517-6524.

[23] E. K. L. Yeow and K. P. Ghiggino, Electronic energy transfer in multichromophoric arrays. The effects of disorder on superexchange coupling and energy transfer rate, J Phys Chem A 104 (2000), 5825-5836.

[24] G. J. Daniels and D. L. Andrews, The electronic influence of a third body on resonance energy transfer, J Chem Phys 116 (2002), 6701-6712.

[25] L. D. Bakalis and J. Knoester, Linear absorption as a tool to measure the exciton delocalisation length in molecular assemblies, $J$ Luminescence 87 89 (2000), 66-70. 
[26] G. D. Scholes and G. R. Fleming, On the mechanism of light harvesting in photosynthetic purple bacteria: B800 to B850 energy transfer, J Phys Chem B 104 (2000), 1854-1868.

[27] S. Gnanakaran, R. Haran, R. Kumble and R. M. Hochstrasser, Energy transfer and localization: applications to photosynthetic systems, Resonance energy transfer (D. L. Andrews and A. A. Demidov, eds), Wiley, New York, NY, USA, 1999.

[28] S. Savikhin, D. R. Buck and W. S. Struve, The Fenna-Matthews-Olsen protein: a strongly coupled photosynthetic antenna, Resonance energy transfer (D. L. Andrews and A. A. Demidov, eds), Wiley, New York, NY, USA, 1999.

[29] H. van Amerongen, L. Valkunas and R. van Grondelle, Photosynthetic Excitons, World Scientific, New Jersey, USA, 2000.

[30] T. Minami, S. Tretiak, V. Chernyak and S. Mukamel, Frenkelexciton Hamiltonian for dendrimeric nanostar, J Luminescence 87-89 (2000), 115118.

[31] A. Archut and G. Vögtle, Functional cascade molecules, Chem Soc Rev 27 (1998), 233-240.

[32] S. Tretiak, V. Chernyak and S. Mukamel, Localized electronic excitations in phenylacetylene dendrimers, J Phys Chem B 102 (1998), 3310-3315.

[33] R. Kopelman, M. Shortreed, Z.-Y. Shi, W. Tan, Z. Xu, J. S. Moore, A. Bar-Haim and J. Klafter, Spectroscopic evidence for excitonic localization in fractal antenna supermolecules, Phys Rev Lett 78 (1997), 1239-1242.

[34] M. Lor, R. De, S. Jordens, G. De Belder, G. Schweitzer, M. Cotlet, J. Hofkens, T. Weil, A. Herrmann, K. Müllen, M. Van Der Auweraer and F. C. De Schryver, Generation-dependent energy dissipation in rigid dendrimers studied by femtosecond to nanosecond time-resolved fluorescence spectroscopy, J Phys Chem A 106 (2002), 2083-2090.

[35] C. Devadoss, P. Bharathi and J. S. Moore, Energy transfer in dendritic macromolecules: molecular size effects and the role of an energy gradient, J Am Chem Soc. 118 (1996), 9635-9644.

[36] M. R. Shortreed, S. F. Swallen, Z. Y. Shi, W. H. Tan, Z. F. Xu, C. Devadoss, J. S. Moore and R. Kopelman, Directed energy transfer funnels in dendrimeric antenna supermolecules, J Phys Chem B 101 (1997), 63186322.

[37] A. Bar-Haim and J. Klafter, Dendrimers as light harvesting antennae, $J$ Luminescence $76 \& 77$ (1998), 197-200.

[38] A. Bar-Haim and J. Klafter, Geometric versus energetic competition in light harvesting by dendrimers, J Phys Chem B 102 (1998), 1662-1664. 
[39] Z. F. Xu and J. S. Moore, Stiff dendritic macromolecules. 4. Design and synthesis of a convergent and directional molecular antenna, Acta Polym 45 (1994), 83-87.

[40] U. Hahn, M. Gorka, F. Vögtle, V. Vicinelle, P. Ceroni, M. Maestri and V. Balzani, Light-harvesting dendrimers: efficient intra- and intermolecular energy-transfer processes in a species containing 65 chromophoric groups of four different types, Angew Chem Int Ed 41 (2002) 3595-3598.

[41] F. Würthner and A. Sautter, Energy transfer in multichromophoric selfassembled molecular squares, Org Biomol Chem 1 (2003) 240-243.

[42] P. G. Van Patten, A. P. Shreve, J. S. Lindsey and R. J. Donohoe, Energytransfer modeling for the rational design of multiporphyrin light-harvesting arrays, J Phys Chem B 102 (1998), 4209-4216.

[43] K. Kilså, J. Kajanus, J. Mårtensson and B. Albinsson, Mediated electronic coupling: Singlet energy transfer in porphyrin dimers enhanced by the bridging chromophore, J Phys Chem B 103 (1999), 7329-7339.

[44] P. Brodard, S. Matzinger, E. Vauthey, O. Mongin, C. Papamicaël and A. Gossauer, Investigations of electronic energy transfer dynamics in multiporphyrin arrays, J Phys Chem A 103 (1999), 5838-5870.

[45] D. Kuciauskas, P. A. Liddell, S. Lin, T. E. Johnson, S. J. Weghorn, J. S. Lindsey, A. L. Moore, T. A. Moore and D. Gust, An artificial photosynthetic antenna-reaction center complex, J Am Chem Soc 121 (1999), 8604-8614.

[46] G. Kodis, P. A. Liddell, L. de la Garza, P. C. Clausen, J. S. Lindsey, A. L. Moore, T. A. Moore and D. Gust, Efficient energy transfer and electron transfer in an artificial photosynthetic antenna-reaction center complex, $J$ Phys Chem A 106 (2002), 2036-2048.

[47] F. Auzel, Upconversion processes in coupled ionic systems, $J$ Luminescence 45 (1990), 341-345.

[48] M. Chua and P. A. Tanner, Three-body energy transfer processes of lanthanide ions in crystals, J Luminescence 66\&67 (1995), 203-207.

[49] X. Zhang, J.-P. Jouart and G. Mary, Energy transfer upconversion in $\mathrm{Ho}^{3+}$ and $\mathrm{Ho}^{3+}, \mathrm{Yb}^{3+}$ doped $\mathrm{CdF}_{2}$ crystals, J Phys: Condens Matter 10 (1998), 493-500.

[50] B. G. Wybourne, Spectroscopic Properties of Rare Earths, Interscience, New York, USA, 1965.

[51] G. H. Dieke, Spectra and Energy Levels of Rare Earth Ions in Crystals, Wiley, New York, USA, 1968. 
[52] V. V. Ovsyankin, Spectroscopy of solids containing rare earth ions (A. A. Kaplyanskii and R. M. Macfarlane, eds), Elsevier, Amsterdam, The Netherlands, 1987.

[53] D. L. Andrews, An accretive mechanism for blue-shifted fluorescence in strongly-pumped systems: resonance energy transfer with Raman emission, J Raman Spectrosc 31 (2000), 791-796.

[54] R. D. Jenkins and D. L. Andrews, Twin-donor systems for resonance energy transfer, Chem Phys Lett 301 (1999), 235-240.

[55] D. L. Andrews and R. D. Jenkins, A quantum electrodynamical theory of three-center energy transfer for upconversion and downconversion in rare earth doped materials, J Chem Phys 114 (2001), 1089-1100.

[56] D. L. Andrews, Anomalous absorption of the ultrafast laser supercontinuum, Phys Rev A 38 (1988) 5129-5139.

[57] S. O. Vasquez, Energy transfer processes in organized media. I. A crystal model for cubic sites, J Chem Phys 104 (1996), 7652-7657.

[58] S. O. Vasquez, Energy transfer processes in organized media. II. Generalization of the crystal model for dipole-dipole interactions in cubic sites, J Chem Phys 106 (1997), 8664-8671.

[59] S. O. Vasquez, Crystal model for energy-transfer processes in organized media: Higher-order electric multipolar interactions, Phys Rev B 60 (1999), 8575-8585.

[60] C. Z. Hadad and S. O. Vasquez, Energy-transfer processes induced by exchange interactions, Phys Rev B 60 (1999), 8586-8594.

[61] L.-S. Lee, S. C. Rand, and A. L. Schawlow, Cooperative energy-transfer among $\operatorname{Pr}^{3+}$ ions in $\mathrm{LaF}_{3}$, Phys Rev B 29 (1984), 6901-6906.

[62] A. Lezama, M. Oriá, J. R. Rios Leite, and Cid B. De Araújo, Triad spectroscopy via ultraviolet upconversion in $\operatorname{Pr}^{3+}-\mathrm{LaF}_{3}$, Phys Rev B 32 (1985), 7139-7142.

[63] J. P. Jouart and G. Mary, Upconversion in $\mathrm{Er}^{3+}$-doped fluorite-type crystals pumped by $1.5 \mu \mathrm{m}$ tunable diode-laser, $J$ Luminescence 46 (1990), 39-45.

[64] G. D. Scholes, X. J. Jordanides and G. R. Fleming, Adapting the Förster theory of energy transfer for modeling dynamics in aggregated molecular assemblies, J Phys Chem B 105 (2001), 1640-1651.

[65] M. Nickoleit, A. Uhl and J. Bendig, Non-linear simultaneous two-photon excitation energy transfer in the wrong direction, Laser Chemistry 17 (1997), 161-174. 
[66] D. M. Junge and D. V. McGrath, Photoresponsive dendrimers, Chem Commun (1997), 857-858.

[67] D. L. Jiang and T. Aida, Photoisomerization in dendrimers by harvesting of low-energy photons, Nature 388 (1997), 454-456.

[68] M. Drobizhev, A. Karotki, A. Rebane and C.W. Spangler, Dendrimer molecules with record large two-photon absorption cross section, Opt Lett 26 (2001), 1081-1083.

[69] R. D. Jenkins and D. L. Andrews, Three-center systems for energy pooling: quantum electrodynamical theory, J Phys Chem A 102 (1998), $10834-10842$.

[70] R. D. Jenkins and D. L. Andrews, Orientation factors in three-centre energy pooling, Phys Chem Chem Phys 2 (2000), 2837-2843.

[71] R. D. Jenkins and D. L. Andrews, Four-center energy transfer and interaction pairs: Molecular quantum electrodynamics, J Chem Phys 116 (2002), 6713-6724.

[72] D. L. Andrews and R. D. Jenkins, Nonlinearities in energy harvesting media, Complex Mediums II: Beyond Linear Isotropic Dielectrics (A. Lakhtakia, W. S. Weiglhofer and I. J. Hodgkinson, eds) Proc SPIE 4467 (2001), 297-306.

[73] R. D. Jenkins, D. L. Andrews and L. C. Dávila Romero, A new diagrammatic methodology for non-relativistic quantum electrodynamics, J Phys B: Atom Molec Opt Phys 35 (2002), 445-468.

[74] R. D. Jenkins and D. L. Andrews, Multichromophore excitons and resonance energy transfer: molecular quantum electrodynamics, $J$ Chem Phys (in press, 2003).

[75] R. D. Jenkins and D. L. Andrews, Exciton energy transfer: effects of geometry and transition moment orientation in model photosystems, Photochem Photobiol Sci (in press, 2003). 